\title{
A method for the accurate numerical prediction of the tip vortices shed from hydofoils
}

\author{
P. Flaszyński, Ph. D. \\ J. A. Szantyr, Prof. \\ R. Biernacki, Ph. D. \\ Gdansk University of Technology \\ P. Dymarski, Ph. D. \\ M. Kraskowski, M. Sc. \\ Ship Design and Research Centre CTO SA
}

\begin{abstract}
The possibly accurate numerical prediction of the detailed structure of vortices shed from the tips of hydrofoils is an important element of the design process of marine propellers. The concentrated tip vortices are responsible for the propeller cavitation erosion and acoustic emission. The purpose of the project described in this paper was to develop the numerical method for prediction of the tip vortex structure. In the course of the project the numerical calculations were confronted with the results of experimental measurements. This led to creation of the specific method of construction of the computational grid and to selection of the optimum turbulence model. As a result the reliable method for the accurate numerical prediction of the concentrated tip vortices for different hydrofoil geometry and flow conditions has been developed and validated. This method enables elimination of the unfavourable phenomena related to the tip vortices in the course of the propeller design calculations.
\end{abstract}

Keywords: marine propulsors, vortex generation, numerical methods, experimental techniques

\section{INTRODUCTION}

The concentrated tip vortices shed from the tips of the marine propeller blades are responsible for many unfavourable phenomena. Due to the strongly reduced pressure in their centres they generate cavitation, which in turn leads to destructive cavitation erosion of the propeller and the rudder, generation of the intensive acoustic signals and induction of vibration in the elements of ship structure (cf. Fig. 1). These phenomena should be accurately predicted at the design stage of the propeller, in order to eliminate them completely or at least to limit their intensity to a harmless level. This can be done by a sufficiently accurate and widely experimentally validated numerical method, which responds correctly to even small modifications of the propeller blade geometry and to small changes in the flow conditions. Such a method may be used for numerical optimization of the marine propeller geometry and its operating conditions.

The first step in numerical prediction of the tip vortex cavitation is the possibly accurate determination of the tip vortex structure, i.e. the spatial distribution of the flow velocity and pressure in the vicinity of the vortex, including its centre. The accurate determination of location and magnitude of the minimum pressure is of particular importance, because this pressure is the decisive factor in cavitation inception. The region in question is dominated by the high velocity transverse flow generated by the vortex. Consequently, the resultant local velocity vectors deviate strongly from the direction of the

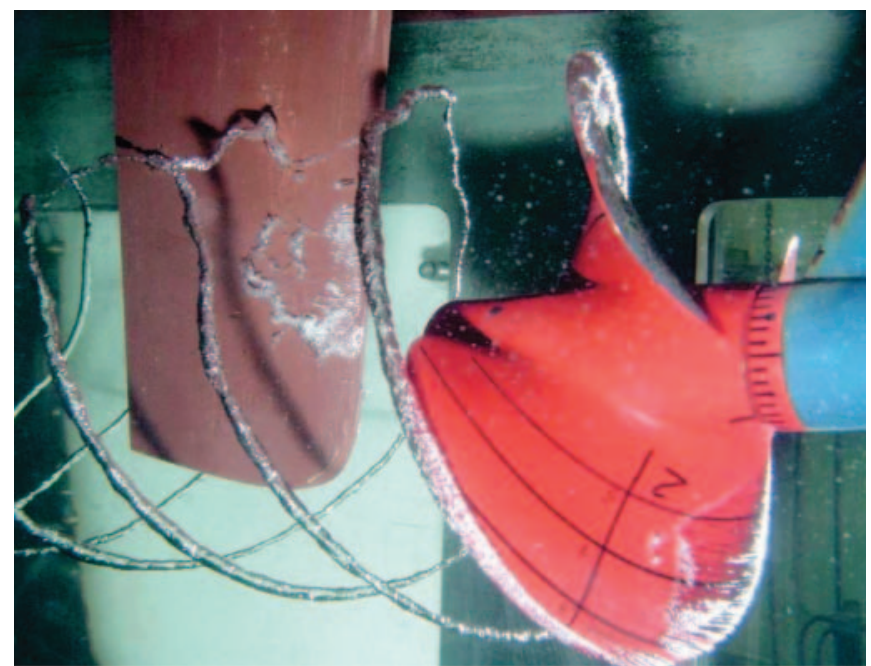

Fig. 1. Cavitating tip vortices shed from the blades of the marine propeller model and interacting with the ship rudder (courtesy of the Ship Design and Research Centre CTO S.A. in Gdansk, Poland)

general flow. This creates special problems for the numerical methods, for example the most frequently used methods based on numerical solution of the Reynolds Averaged Navier Stokes equations. These problems concern the adequacy of the computational grid structure and the accuracy of the turbulence models, which have been calibrated for much simpler flows. The accumulated practical experience of the past shows, that 
in most cases the numerical methods tend to underestimate the degree of concentration and the minimum pressure of the tip vortices. Consequently, their ability to predict accurately the tip vortex cavitation inception was regarded as questionable.

The purpose of the research project described in this paper was to develop a computational method, which makes use of the special structure of the computational grid and which employs the specific turbulence models, especially suited for the vortexdominated flows. This method is based on the combined numerical and experimental research, presented in detail in $[1,2,3]$ and described briefly in the following section.

\section{DESCRIPTION OF THE NUMERICAL AND EXPERIMENTAL RESEARCH}

The research forming the basis for the development of the new method included experiments [4] and calculations [5, 6, 7, 8, $9,10]$. The experiments were conducted in the cavitation tunnel of CTO S.A. and they were concerned with the measurements of the velocity field in three cross-sections of the tip vortex generated by hydrofoils of different geometry in different flow conditions (cf. Fig. 2). The measurements were performed using the Laser Doppler Anemometer. The geometry of the hydrofoils was selected in such a way that it resembled the blades of contemporary marine propellers. Three variants of the hydrofoil were tested: one with optimum distribution of the hydrodynamic loading along its span, one with hydrodynamically unloaded tip and one with hydrodynamically loaded tip. Each of these hydrofoils was tested at three different angles of attack: +2.5 degrees, 0 and -2.5 degrees. It was expected that the variation of the hydrodynamic loading along the span of the hydrofoils may influence the process of formation of the tip vortices and it may lead to generation of vortices of different strength. The sufficiently accurate numerical method should be able to detect these small differences.

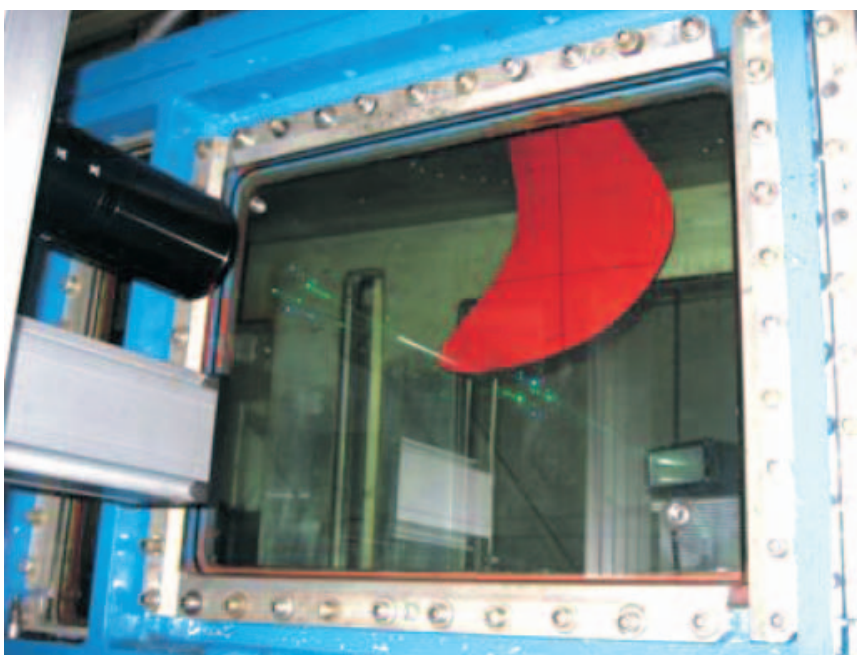

Fig. 2. Hydrofoil model mounted in the measuring section of the cavitation tunnel of CTO S.A.

The numerical calculations were arranged in such a way that they reproduced the experiments as closely as possible. The details of the computational flow domain, based on the geometry of the measuring section of the cavitation tunnel, are shown in Fig. 3.

The calculations were performed using three different CFD codes (commercial codes Fluent and Comet together with the code Solaga developed at CTO S.A.) and in all six turbulence models (Spalart-Allmaras, k- $\omega, \mathrm{k}-\varepsilon$, RSM, k- $\omega$ SST, $\mathrm{k}-\varepsilon \mathrm{RNG})$. The accuracy of the results of calculations for such a complicated, vortex-dominated flow, depends on the detailed structure of the computational grid and on the adequacy of the applied turbulence models. The purpose of calculations was to develop a method for construction of the optimum grid for this specific type of flow and to select a turbulence model which is best suited to simulate the process of turbulence generation, transport and dissipation in this type of flow. This was performed on the basis of comparison of the experimental and computational results in three cross-sections of the vortex, located respectively $10 \mathrm{~mm}, 70 \mathrm{~mm}$ and $300 \mathrm{~mm}$ behind the hydrofoil tip, as shown in Fig. 4.
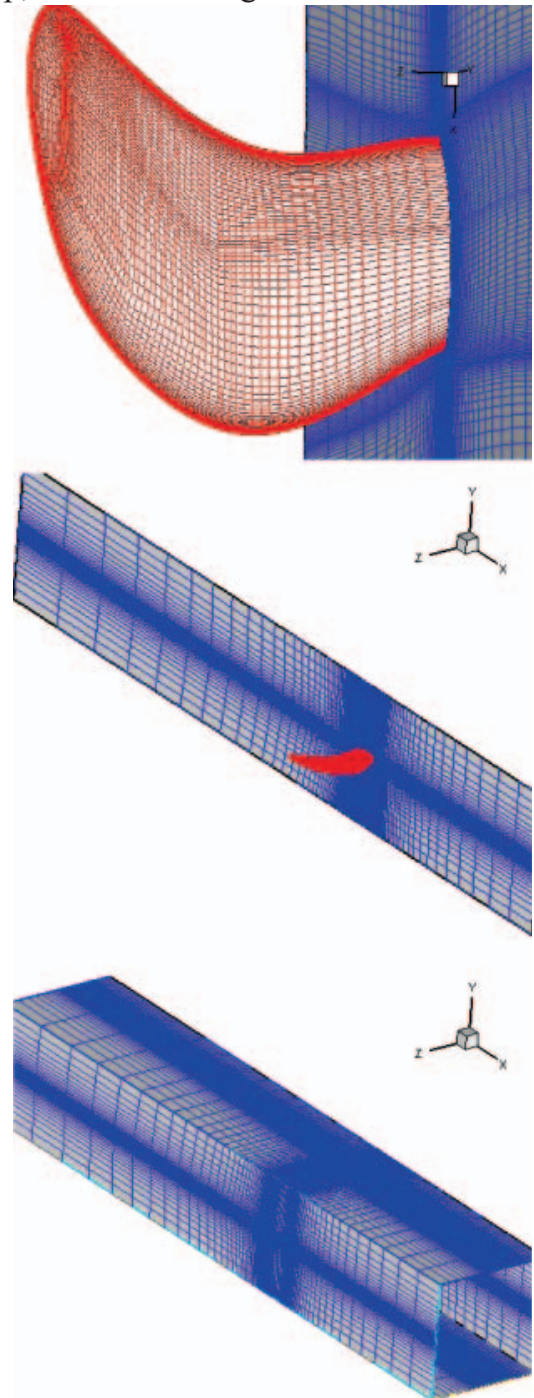

Fig. 3. The discrete mesh for numerical calculations on the hydrofoils surface (top) and on the external surfaces of the flow domain (bottom)

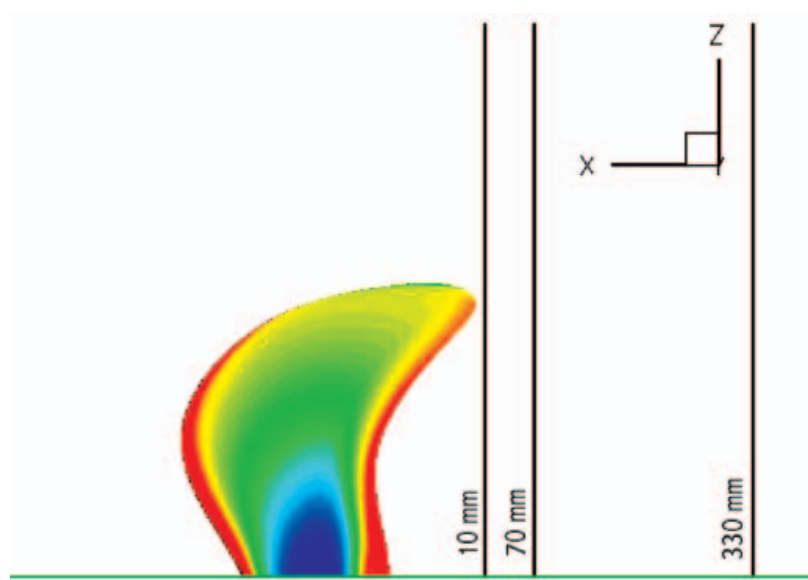

Fig. 4. Location of the cross-sections of the tip vortex for computations and measurements 
The process of development of the optimum computational grid was conducted in three stages:

- the first, basic grid was constructed according to the general recommendations included in the manuals of the respective CFD codes (cf. Fig. 5). This was a block-structured grid, in which the O-type grid was employed around the hydrofoil and the $\mathrm{H}$-type grid was used in the remaining part of the flow domain. The total number of finite volumes was around 1.250.000 and the size of the smallest volume close to the surface of the hydrofoil was about $0.02 \mathrm{~mm}$.

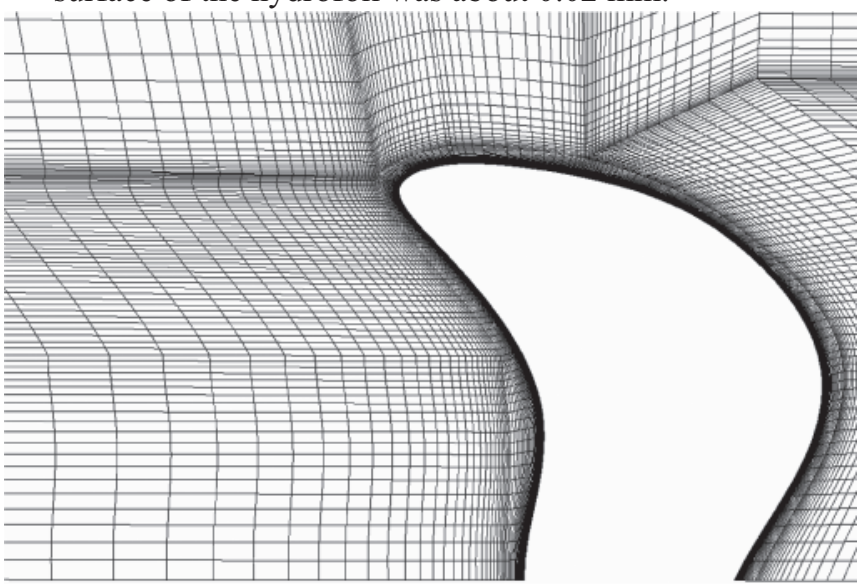

Fig. 5. Basic computational grid in the vicinity of the hydrofoil

- the second grid was refined inside the cylinder covering the anticipated location of the tip vortex (cf. Fig. 6). Inside this cylinder all initially defined finite volumes were divided into 8 smaller volumes, rising the total number of volumes to about 1.940 .000 .

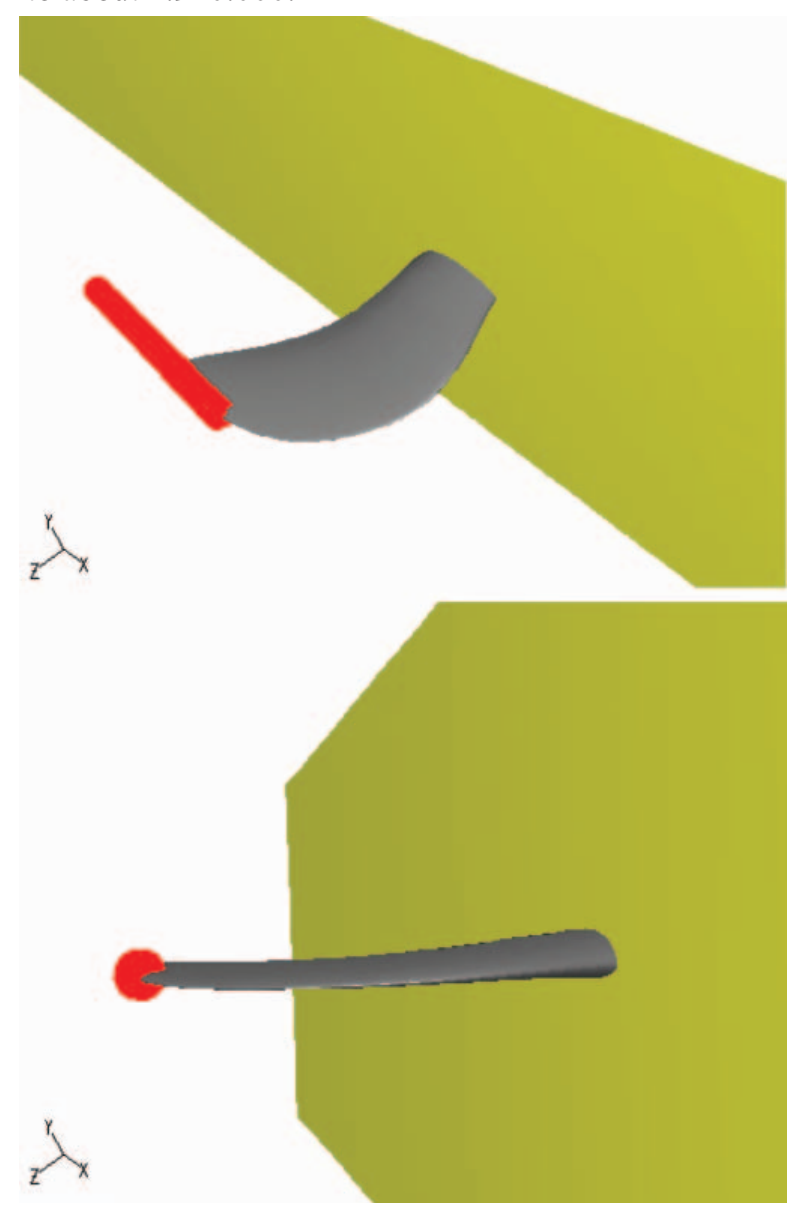

Fig. 6. Computational grid refined in an arbitrarily defined cylinder
- the third grid was refined in the semi-automatic way, using the criterion based on the local values of vorticity, as calculated using the basic grid (cf. Fig. 7). The values of vorticity were tested inside the blue region shown in Fig. 7 and the volumes inside the red region in which the vorticity exceeded the prescribed value were modified. Each finite volume in the modified region was divided into 16 smaller volumes, rising the total number of volumes to about 1.638.300. In order to have the refined grid in the tip vortex (core and vicinity), the streamwise voriticity has been selected as the criteria. The range of the streamwise vorticity for the grid adaptation is case dependent. In general, one can apply the maximum of the vorticity as the upper limit and $30 \%$ of the maximum with the opposite sign as the lower limit. The lower limit is also important, because the counter rotating vortices are generated downstream the trailing edge in some cases. They are much weaker than the main vortex, but their influence on the tip vortex development and the diffusion rate is important. In case of the Comet code, the streamwise velocity was applied as the criteria for the grid adaptation.
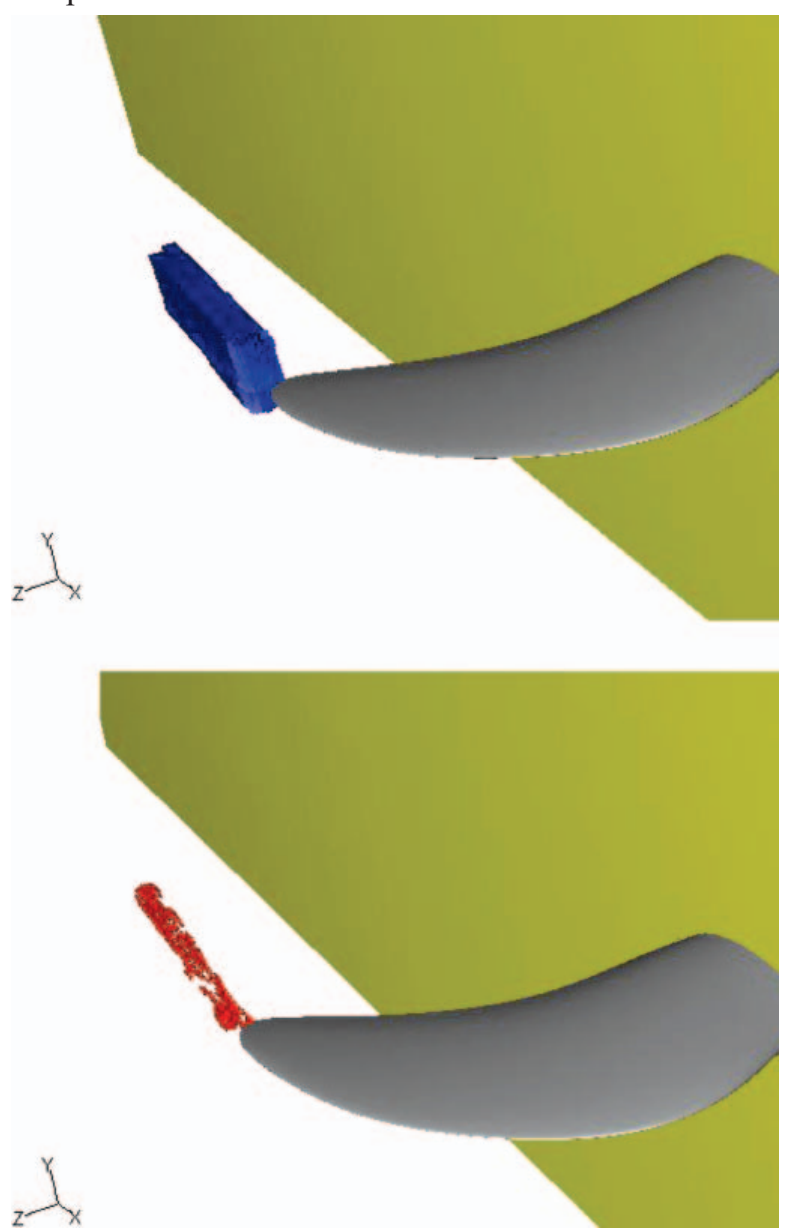

Fig. 7. Computational grid refined on the basis of the local values of vorticity

The distributions of the $\mathrm{x}$-component of the resultant flow velocity and the $\mathrm{x}$-component of the local vorticity calculated using the first grid, which were later used in the semi-automatic grid refinement, are shown in Fig. 8. In the same Figure the resulting grid structures in the consecutive stages: basic grid, adaptation 1 and adaptation 2 are shown.

It is easy noticeable that the basic grid is coarse. Such effect could be avoided if the tip vortex location could be foreseen during grid generation and the grid would be generated fine enough at the very beginning. It is difficult or even impossible 

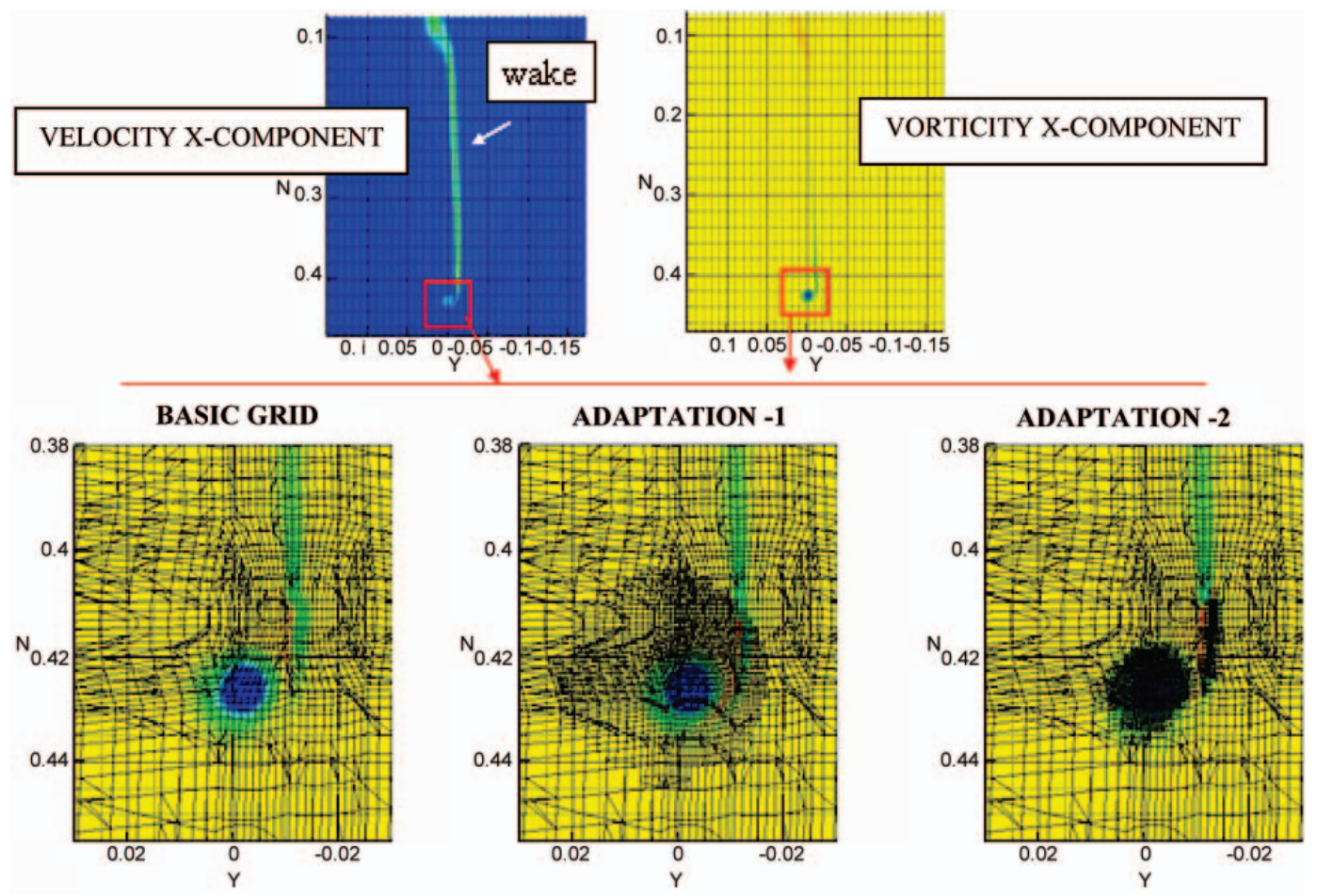

Fig. 8. Process of grid refinement in the tip vortex area

for the full 3D propeller analysis. In such a case, the coarse grid can be created and then it can be refined according to the local flow conditions. The local grid refinement (shown in Fig. 8) enables much better prediction of the local velocity and pressure gradients, which are very important for the cavitation prediction. It is shown that grid adaptation gives higher cells concentration in the tip vortex and such approach seems to be the most efficient.
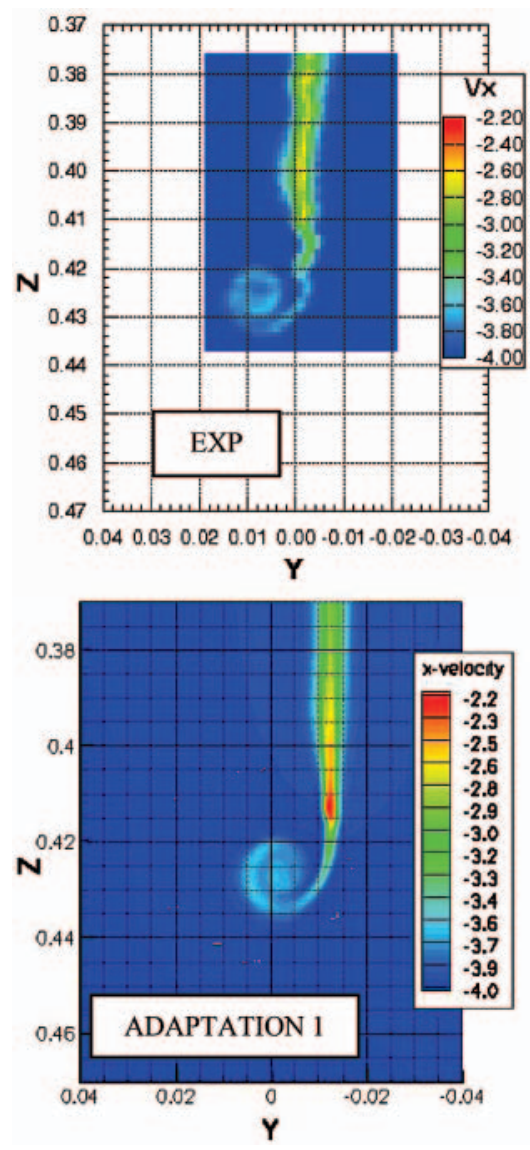

Fig. 9. Axial component of the velocity $-10 \mathrm{~mm}-L+2.5-$ Fluent (SST)

\section{ANALYSIS OF THE NUMERICAL AND EXPERIMENTAL RESULTS}

The selected results of measurements and calculations included in this section are supposed to illustrate the agreement between them for the three consecutive stages of the grid refinement. Fig. 9 presents the axial velocity component in the cross-section $10 \mathrm{~mm}$ behind the hydrofoil, obtained
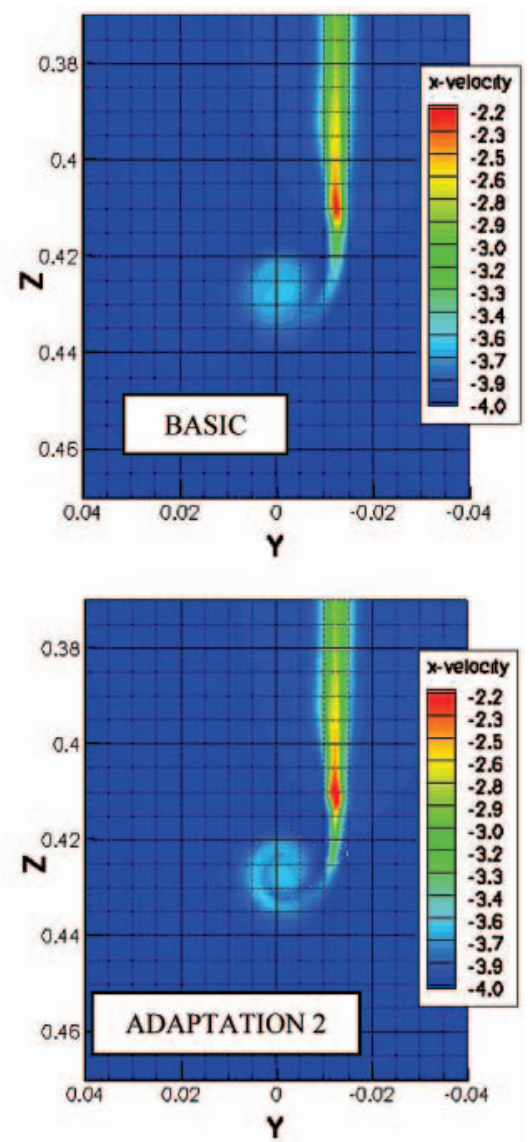
experimentally and calculated using Fluent for the tip-loaded hydrofoil (L) at the angle of attack +2.5 degrees. This hydrofoil geometry in combination with the flow conditions should generate the most intensive vortex in the entire project. Fig. 10

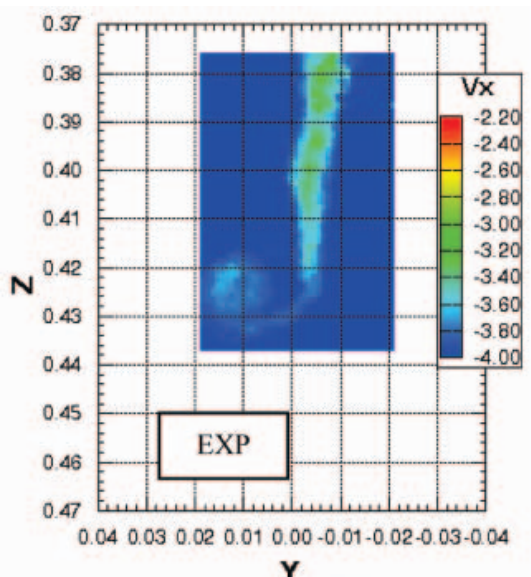

$\mathbf{Y}$

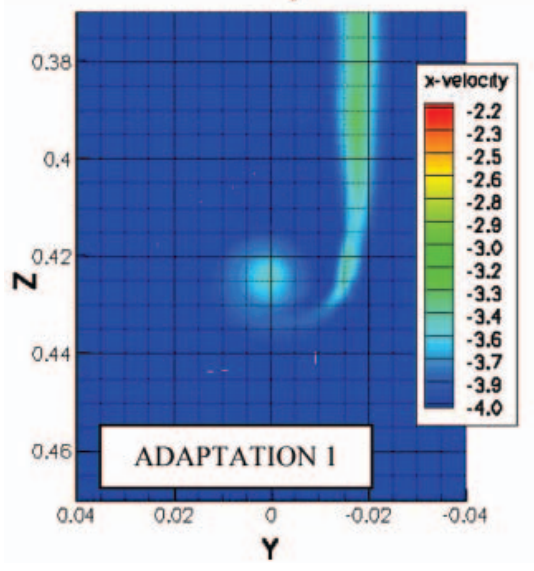

Fig. 10. Axial component of the velocity $-70 \mathrm{~mm}-L+2.5-$ Fluent (SST)
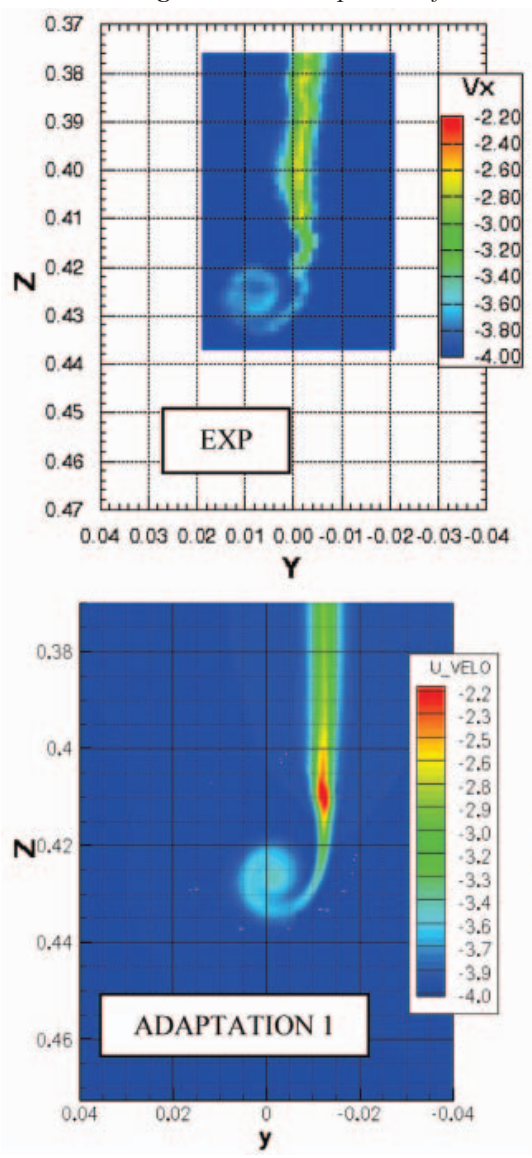

shows the analogical results for the cross-section $70 \mathrm{~mm}$ behind the hydrofoil. Figs. 11 and 12 show similar results obtained by the program Comet. Figs 13 and 14 include the comparison of the experimental and computational results for the transverse
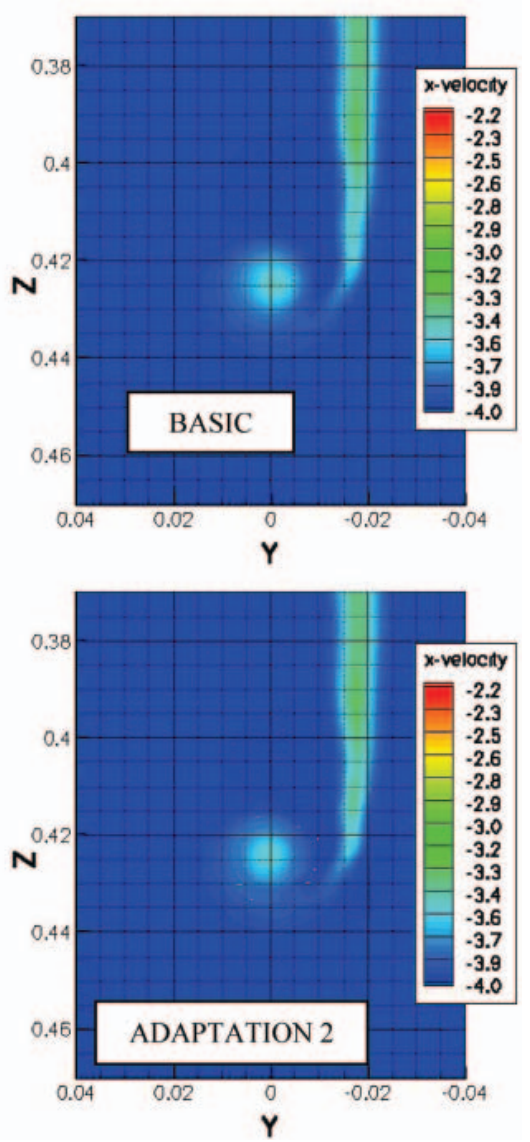
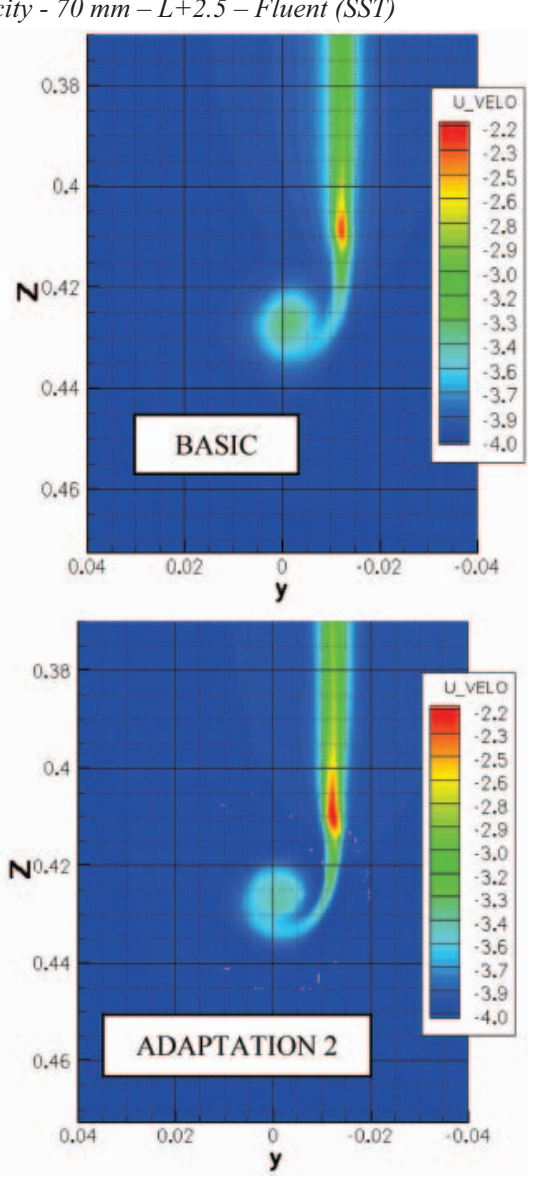

Fig. 11. Axial component of the velocity $-10 \mathrm{~mm}-L+2.5-$ Comet (SST) 
(vertical) velocity component at the cross-section $10 \mathrm{~mm}$ behind the tip-loaded hydrofoil at +2.5 angle of attack.

In all cases computed by Fluent, the best agreement with experimental data was obtained for the "adaptation-2". In the
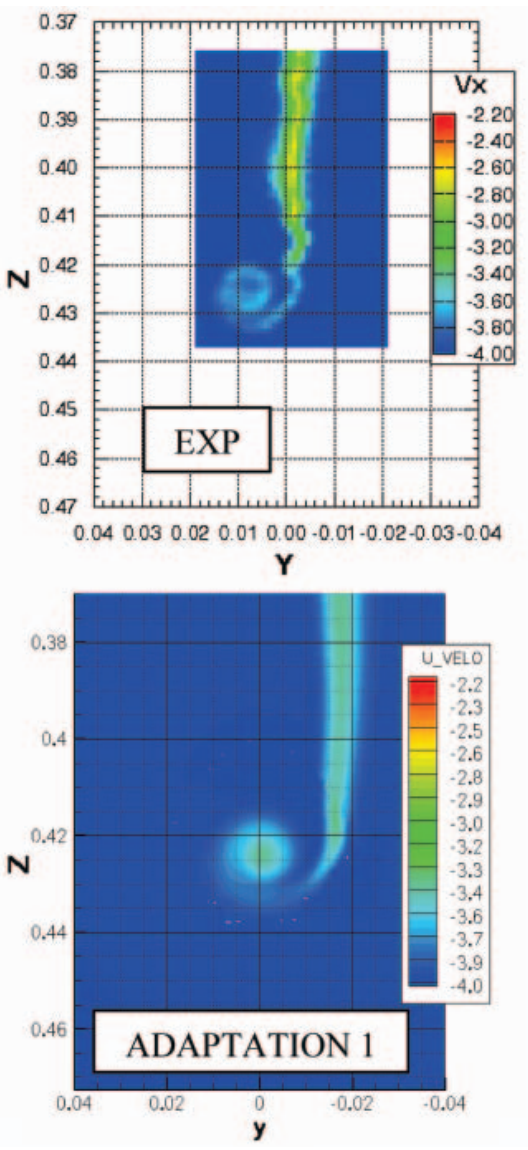

Fig. 12. Axial component of the velocity - $70 \mathrm{~mm}-L+2.5-$ Comet (SST)
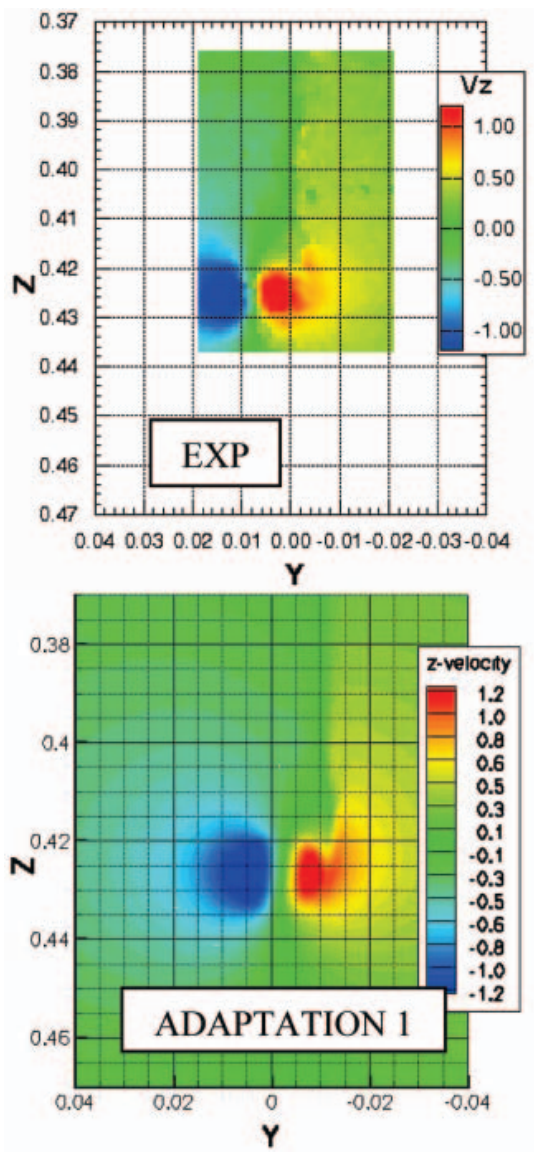

Comet code, because it was no possibility to set the vorticity as the grid refinement criterion, the longitudinal velocity was applied. For such a criterion, which is not so sensitive as the vorticity, the results obtained on the refined grids differ
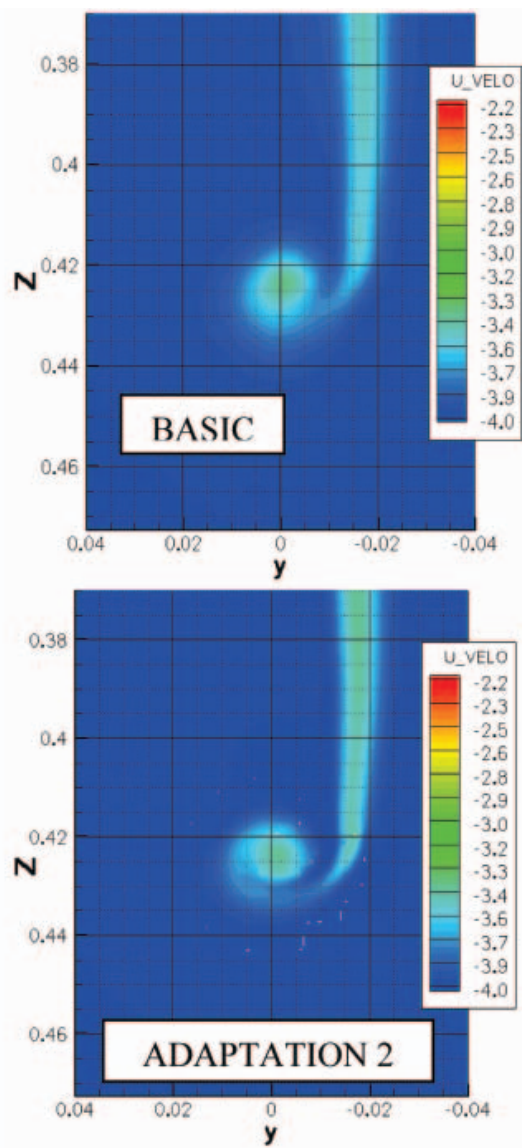
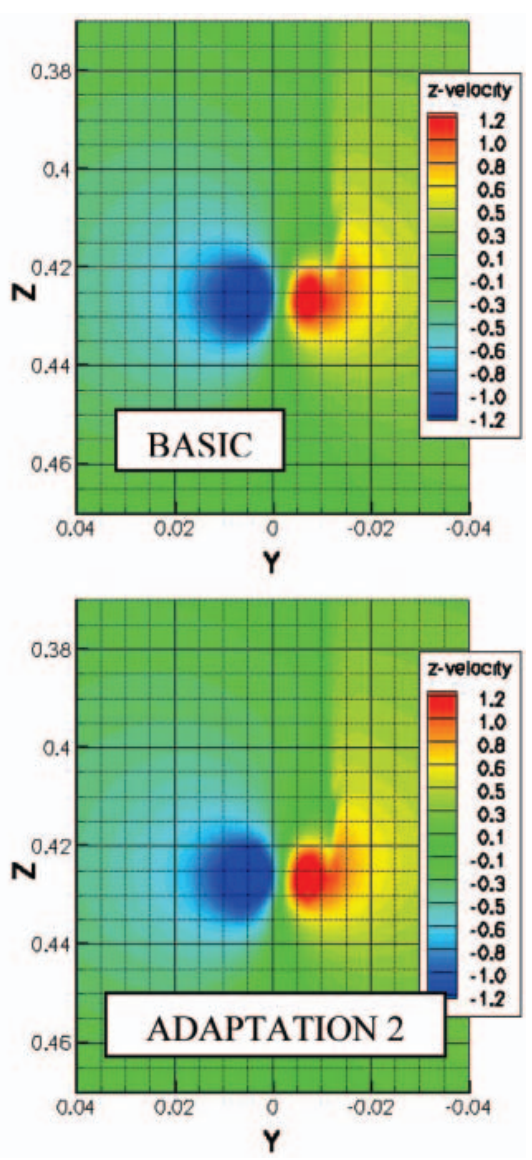

Fig. 13. Vertical component of the velocity $-10 \mathrm{~mm}-L+2.5-$ Fluent (SST) 

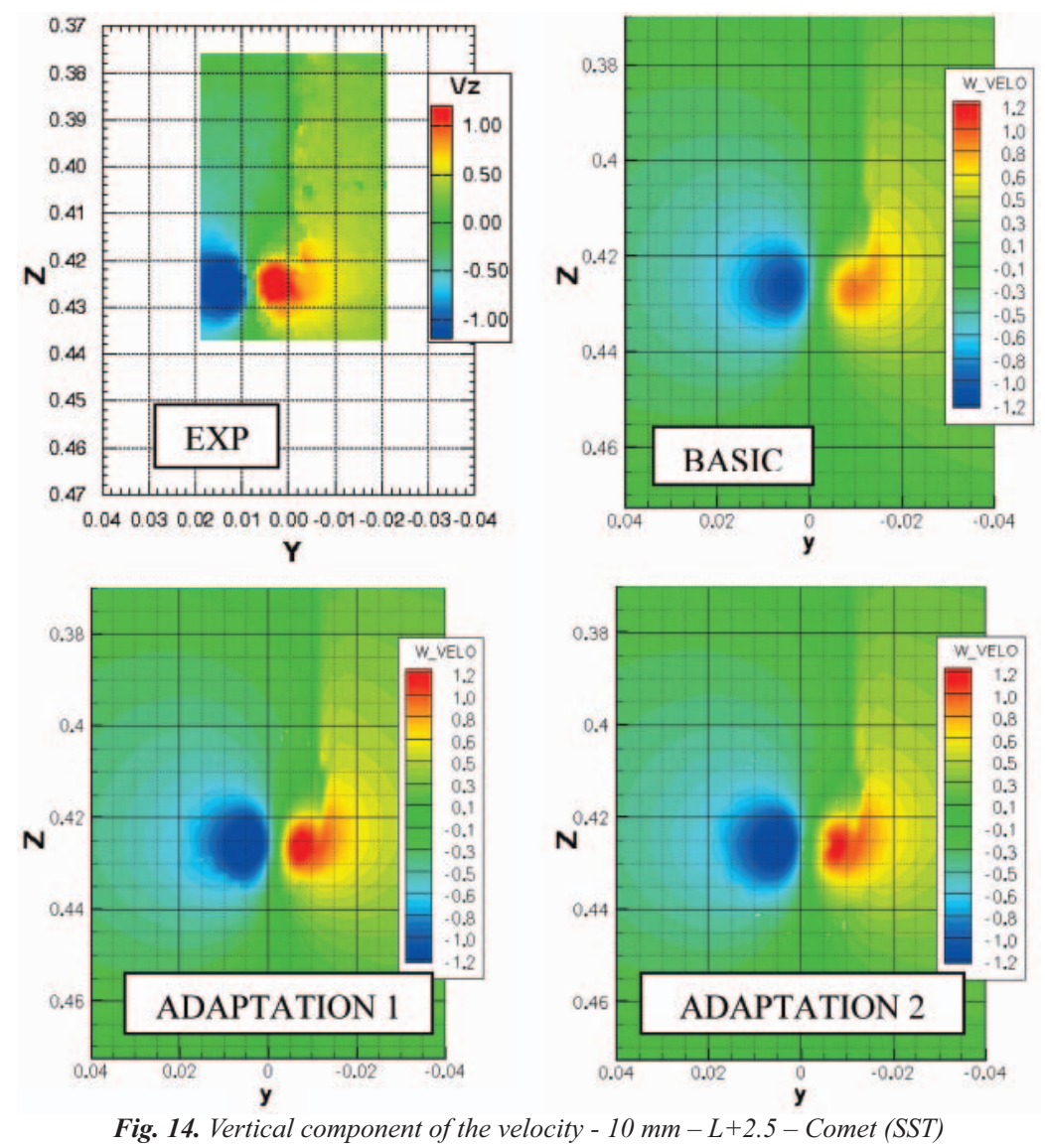

from those obtained with Fluent. In some cases (Fig.1112), the results obtained on the grid for the first adaptation (adaptation-1) are a bit closer to the measurements.

\section{METHOD FOR NUMERICAL PREDICTION OF TIP VORTICES}

The method developed and tested in the course of the described research project is in fact the method for effective and optimum use of the commercial CFD codes for the specific task of accurate prediction of the tip vortices generated by hydrofoils. This method is built on two basic recommendations:

- the computational grid may be initially constructed according to general rules applicable to the CFD code used. After performing the initial calculations using this grid, the region of the anticipated location of the tip vortex must be defined and the grid in this region should be modified (subdivided at least by a factor of 16), using the criterion based on the calculated $\mathrm{x}$ (streamwise) component of the local flow vorticity. Then the calculation should be repeated using the modified grid, producing the final results.

- in the RANS methods applied for the vortex-dominated flows the turbulence model k- $\omega$ SST should be used, because it is best suited for such flows and it gives the best results as far as the prediction of the detailed vortex structure is concerned.

These recommendations have been proven to be effective for two different commercial CFD codes used in the project. Therefore it may be safely assumed that they are valid also for other commercial codes. Calculations of the tip vortices generated by hydrofoils, which are conducted in accordance with the above recommendations, supply sufficiently accurate results for further analysis of the tip vortex cavitation.

\section{CONCLUSIONS}

The following final conclusions may be formulated on the basis of the research presented above and in [3]:

- contemporary Computational Fluid Dynamics methods are fully capable of predicting the vortex-dominated flows with accuracy sufficient for further analysis of the vortex cavitation inception and development

- the accuracy of numerical prediction of the vortexdominated flows is significantly improved by the semiautomatic refinement of the computational grid in the region of the vortex, based on the pre-computed values of the local axial (streamwise) component of vorticity

- such a local refinement of the computational grid ensures acceptable accuracy of the results while keeping the calculation time to a reasonable minimum

- in the sufficiently dense grid (in both directions: crosswise and streamwise) the diagonal orientation of the resultant velocity vectors does not influence adversely the results of calculations of the vortex-dominated flow

- among the most popular turbulence models the k- $\omega$ SST model seems to be best suited for calculations of the vortexdominated flows.

\section{Acknowledgement}

The research presented in this paper has been conducted with the support of the Research Grant no. N504 033 21/2951 of the Polish Ministry of Science and Higher Education 


\section{BIBLIOGRAPHY}

1. Dymarski P., Szantyr J., Flaszyński P., Kraskowski M., Biernacki R.: Modelling of Tip Vortex Behind a Blade Using Different Turbulence Models and Different RANSE Solvers. Comparison with LDA Measurements, Proc. of the 11th Numerical Towing Tank Symposium, Brest, France, September 8-10 2008

2. Flaszyński P., Szantyr J., Dymarski P., Krasowski M.: Numerical Prediction of Vortex Generated by a Hydrofoil, Proc. of the International Symposium on Marine Propulsors, Trondheim, Norway, June 22-24, 2009

3. Flaszyński P., Szantyr J., Biernacki R., Dymarski P., Kraskowski $\mathrm{M}$, : An Experimental and Numerical Study of the Vortices Generated by Hydrofoils, Polish Maritime Research Vol. 16, No. 4 (63), 2009

4. CTO S.A. Report No. RH-2008/T-133 Results of measurements of the velocity field in the vortex path behind the hydrofoils (in Polish)

5. CTO S.A. Report No. RH-2008/T-019 Calculations for the first variant of the discrete mesh - program Fluent (in Polish)

6. CTO S.A. Report No. RH-2008/T-020 Calculations for the first variant of the discrete mesh - programs Comet and Solaga (in Polish)

7. CTO S.A. Report No. RH-2008/T-110 Calculations for the second variant of the discrete mesh - program Fluent (in Polish)
8. CTO S.A. Report No. RH-2008/T-111 Calculations for the second variant of the discrete mesh - program Comet (in Polish)

9. CTO S.A. Report No. RH-2009/T-019 Calculations for the third variant of the discrete mesh - program Comet (in Polish)

10.CTO S.A. Report No. RH-2009/T-019 Calculations for the third variant of the discrete mesh - program Comet (in Polish)

\section{CONTACT WITH THE AUTHORS}

P. Flaszyński Ph. D. e-mail: pflaszyn@pg.gda.pl J. A. Szantyr Prof. e-mail: jas@pg.gda.pl

R. Biernacki Ph. D., e-mail: rbiernac@pg.gda.pl Faculty of Mechanical Engineering Gdansk University of Technology Narutowicza 11/12 80-231 Gdansk

P. Dymarski Ph. D., e-mail: padym@cto.gda.pl M. Kraskowski M. Sc, e-mail: Marek.Kraskowski@cto.gda.pl Ship Design and Research Centre CTO S.A. Wały Piastowskie 1 80-958 Gdansk 